\title{
Kernos
}

Revue internationale et pluridisciplinaire de religion grecque antique

$11 \mid 1998$

Varia

\section{Panthéons locaux de Lycie, Lykaonie et Cilicie aux deuxième et premier millénaires av. J.-C.}

\section{René Lebrun}

\section{OpenEdition \\ Journals}

\section{Édition électronique}

URL : http://journals.openedition.org/kernos/1223

DOI : 10.4000/kernos. 1223

ISSN : 2034-7871

\section{Éditeur}

Centre international d'étude de la religion grecque antique

\section{Édition imprimée}

Date de publication : 1 janvier 1998

ISSN : 0776-3824

\section{Référence électronique}

René Lebrun, « Panthéons locaux de Lycie, Lykaonie et Cilicie aux deuxième et premier millénaires av. J.-C. », Kernos [En ligne], 11 | 1998, mis en ligne le 21 avril 2011, consulté le 19 avril 2019. URL : http:// journals.openedition.org/kernos/1223; DOI : 10.4000/kernos.1223 


\section{Panthéons locaux de Lycie, Lykaonie et Cilicie aux deuxième et premier millénaires av. J.-C.}

Bons héritiers de ce que nous révèlent les sites préhistoriques de Çatal Höyük ou d'Haçilar, ou, à une date plus proche de l'installation des tribus indo-européennes en Anatolie, le site d'Alaça Höyük, les panthéons locaux d'Asie Mineure montrent, au second millénaire avant notre ère, une structure relativement homogène et bien identifiable à l'heure présente; celle-ci se retrouve au niveau du panthéon impérial d'après les listes officielles de divinités mentionnées par exemple à la fin des traités ou d'après les reliefs rupestres du célèbre sanctuaire de Yazĭlîkaya situé à environ un kilomètre et demi de la capitale Hattusa et aménagé ou achevé durant la seconde moitié du xirI ${ }^{e}$ siècle av. J.-C. par le roi Tudhaliya IV.

A. La structure des panthéons locaux se hiérarchise comme suit :

1. Dieu de l'orage associé à une divinité solaire masculine ou féminine, ou à une déesse mère;

2. Divinité protectrice de la nature sauvage;

3. Éventuellement un dieu de la guerre;

4. Forces chthoniennes comme les montagnes, les sources et les rivières.

Cette structure de base est clairement évoquée dans des recensions de panthéons locaux, mais elle se trouve également figurée sur certains sceaux ${ }^{1}$.

1 Cf. par exemple la tablette KBo XII 135 ( 4 colonnes au recto, 4 colonnes au verso), époque tardive; on observe régulièrement le schéma : dieu de l'orage, divinité solaire, dieu protecteur de la nature sauvage et une montagne; il en est ainsi pour Sarusna, Sekta; à Ibbitta, nous trouvons une déesse reine à la place de la montagne ou de la source. Le panthéon de Hubesna $=\mathrm{cl}$. Kybistra est un peu plus complexe puisqu'il donne la séquence divine suivante: tout d'abord, Huwassanna, la déesse reine, suivie du Soleil, du dieu de l'orage, du dieu protecteur de la nature sauvage, du mont Sarlaimi = « Sublime ", à la suite duquel sont mentionnés le dieu de la guerre, Lallariya et le mont Sarpa, et enfin quelques dieux mineurs. On trouvera aussi une intéressante liste des panthéons locaux hittites à l'époque du roi Muwatalli II, lequel régnait à la fin du xive-début du xiII ${ }^{\mathrm{e}}$ siècle av. J.-C., dans la grande prière qu'il adresse à tous les dieux (CTH 381), dont une excellente édition vient d'être publiée par I. Singer, Muwatalli's Prayer to the Assembly of Gods Through the Storm-God of Lightning, Atlanta, 1996; évoquons simplement le panthéon de la métropole religieuse de haute antiquité qu'était Arinna au Hatti central, non loin de Hattusa : dieu Soleil des cieux, déesse Solaire d'Arinna, dieu de l'orage d'Arinna, Mezzulla (fille de la déesse Soleil d'Arinna), Hulla, Zintuhi, dieux masculins, déesses, montagnes et rivières d'Arinna, dieu de l'orage du salut, dieu de l'orage de la vie (ces 
Dans de nombreux cas, on relèvera l'absence de dieu Lune, dont tout laisse à penser cependant qu'il fut particulièrement vénéré en Anatolie méridionale. D'après les données actuelles, les catégories divines que nous venons d'énoncer, s'appliquaient aux panthéons locaux de Lycie, Pamphylie, Lykaonie, Pisidie et Cilicie.

B. En considérant plus particulièrement la côte méditerranéenne et les régions avoisinantes, de culture louvite, nous constatons aujourd'hui que plusieurs centres religieux célèbres à l'époque gréco-asianique existaient bel et bien au deuxième millénaire; leurs panthéons respectifs ainsi que leur vie cultuelle sortent progressivement de l'ombre. Citons pour mémoire d'Ouest en Est : Xanthos, Tlôs, Pinara et Oinoanda en Lycie, Pergè en Pamphylie, Lystra en Lykaonie, Comana en Cataonie, et Tarse en Cilicie ${ }^{2}$. Le problème

deux derniers étant des hypostases du dieu de l'orage hourrite Teshub; il s'agit donc d'une addition d'époque impériale) $=$ texte A I 37-39. Mentionnons encore le panthéon de Nenassa : dieu de l'orage de Nenassa, Lusiti de Nenassa, le fleuve Marassantiya (= Halys), les dieux masculins, les déesses, les montagnes (et) les rivières de Nenassa = texte A II 12-14. De plus, une intéressante liste de dieux locaux peut être trouvée dans KBo IX $98+$ KUB LX 46 + KUB LVII 87 analysée par SINGER, op. cit., p. 165-166. Les cultes de chaque ville hittite sont également évoqués dans J. Tischler et G.F. DEL Monte, Répertoire géograpbique des textes cunéiformes (abrév. RGTC), VI, Wiesbaden, 1978, et supplément paru en 1992. Il n'est pas exclu que le panthéon local d'une cité se trouve représenté sur certains sceaux; signalons, par exemple, les quatre côtés d'un sceau cubique représentant respectivement un dieu assis sur son trône, un cerf, un taureau et un lion, $c f . R . M$. Bozhmer et H,G. Güterbock, Glyptik aus dem Stadtgebiet von Boğazköy, Berlin, 1987, p. 56 et table XV fig. 148. Signalons encore la mention d'un panthéon local ou semiofficiel sur les autels trouvés à Emirgazi (région de Konya; règne du roi Tudhaliya IV) où par deux fois ( $(26$ et 29 ) on rencontre la séquence suivante : déesse Soleil d'Arinna, dieu de l'orage des cieux, dieu cerf, le dieu mons.mEnsa, une déesse.

2 Xanthos, en lycien Arñna, pourrait correspondre à une des villes dénommées Arinna dans les textes hittites, ou plus probablement à la cité d'Awarna des tablettes hittites = Awarna du bloc 13 de l'inscription louvite hiéroglyphique de Yalburt (règne de Tudhaliya IV) identifiée avec Xanthos par M. PоEтTo, L'iscrizione Luvio-geroglifica di Yalburt, Pavie, 1993 (Studia Mediterranea, 8), p. 78; Tlôs, en lycien Tlawa, correspond au Tlawa des textes hittites et louvites du second millénaire et on relèvera spécialement la mention de Tlawa dans le bloc 14 de l'inscription de Yalburt; Pinara, lycien Pinale, se trouve probablement mentionnée en KUB XIX 55 et dans les blocs 12 et 13 de l'inscription de Yalburt : pi-na- L.416 (URBS); Oinoanda de Lycie doit correspondre au toponyme Wiyanawanda de certains textes hittites et de l'inscription louvite hiéroglyphique du Südburg de Boğazköy (règne de Suppiluliuma II) $\$ 1$ b ainsi que du bloc 9 de l'inscription de Yalburt, $c f$., pour ces toponymes lyciens, PoETTo, op. cit., p. 79-82; J.D. Hawkins, The Hieroglyphic Inscription of the Sacred Pool Complex at Hattusa (Südburg), Wiesbaden, 1995, p. 29 et 49. La ville de Pergè correspond à la cité de Parha mentionnée dans la fameuse tablette de bronze du traité conclu entre le roi hittite Tudhaliya IV et son vassal Kurunta, roi de Tarhuntassa ( $c f$. édition et étude du document par H. OtTen, Die Bronzetafel aus Boğazköy, Wiesbaden, 1988 (StBot, Beiheft 1), 93 p., 3 pl. et copies autographes). Les panthéons de Lystres et d'Ikonium (Konya) sont encore mal connus pour la période gréco-asianique, mais en revanche nous savons pertinemment qu'Ikonium était une ville importante au second millénaire et se dénommait Ikkuwaniya ( $c f$. textes de 
consiste donc à évaluer l'héritage éventuel des cultes gréco-asianiques à partir de ce que nous savons de ces mêmes sites dans le domaine religieux au deuxième millénaire avant notre ère. En d'autres termes, existe-t-il une solution de continuité en cette matière, ou, si l'on préfère, les Zeus Tarsios, Solymeus ou Kôrykios continuent-ils le Tarchunt/Tarchuna louvite distinct du Zeus olympien grec? Interrogeons-nous encore sur la permanence du culte de certains dieux indigènes jusqu'à l'époque gréco-asianique.

C. La réponse à un tel problème implique d'abord de rappeler qu'à la dislocation de l'Empire hittite vers 1180 av. J.-C., la civilisation anatolienne se poursuit, sous la forme louvite, notamment dans le Sud-Est anatolien et en Syrie du Nord jusque vers 700 av. J.-C., au sein de petits royaumes dont les destins furent diversifiés: Tabal, Kargémish, Hilakku/Qué, Hamath, Adana, par exemple. Après 700, les sources en langue anatolienne (louvite) se taisent pour ces régions. D'autre part, dans le Sud-Ouest anatolien, il n'existe, à ce jour, aucun témoignage indigène après 1050 av. J.-C. jusqu'au ve siècle avant notre ère, lorsque, notamment dans le cadre de l'Empire achéménide, apparaissent de nombreux documents épigraphiques en lycien, ou encore en lydien, en carien, en pisidien, et ceci à côté des documents en grec, en araméen ou parfois bilingues.

D. Examinons quelque peu le cas lycien. À Tlôs (Tlawa) dont les documents hittites nous apprennent l'existence au deuxième millénaire, nous observons, grâce à deux longues inscriptions en langue lycienne, un panthéon dominé par Trqqñt $=$ louvite Tarchunt $=$ Zeus (anatolien) associé à la ẽni qlabi ebijebi « la Mère du sanctuaire d'ici » assimilée à Létô dans le cadre d'un syncrétisme gréco-anatolien. Cette sorte de Magna Mater lyçienne se dénommera Qebeliya à Limyra (lyc. Zêmuri) ou portera encore le nom caractéristique de ẽni mahanabi « mère des dieux » prolongeant le louvite anni- massanassi- ${ }^{3}$. En de nombreux endroits, l'antique divinité anatolienne Maliya s'affirme comme la divinité poliade, ce qui justifie son assimilation à Athéna dans les textes bilingues. Mais il convient de bien identifier Maliya comme une déesse protectrice de ces éléments naturels que sont les vergers,

Boğazköy, inscription du Südburg $₫ 1,4$ ) tandis que Lystres s'appelait Lusna à l'époque hittite et son panthéon se composait d'un dieu Soleil louvite (probablement Tiwaliya), d'un dieu de l'orage, d'une divinité protectrice de la nature sauvage et de quelques divinités indigènes mal connues à ce jour, $c f$. KBo VII 66 III 6' sq. et KUB XVII 19 I 9'. La cappadocienne Comana correspond manifestement à la ville sainte de Kummanni au deuxième millénaire (cf. H.M. Kümmes, Kummanni, in RlAss., 6 Band [1980-1983], p. 335336.), et le grec Tarsos/latin Tarsus rendait le hittite Tarsa.

3 Cf. TL 102, 3 : qebeliya: êni : qlabi: ebij [e] bi: pñtreñni; à propos de ce théonyme issu du nom divin hittito-louvite Hapaliya-, $f$. R. Lebrun, Continuité cultuelle et religieuse en Asie Mineure, in Atti del II Congresso internazionale di Hittitologia, Pavie, 1995 (Studia Mediterranea, 9), p. 250-251. Pour èni mabanabi, cf. TL 134, 4. 
les fleurs et les ruisseaux ${ }^{4}$; elle est donc une divinité relevant de la seconde catégorie, comme le sont sans doute les diverses Artémis locales, celles de Komba, de Lagbè et de Pergè figurant parmi les plus typiques ${ }^{5}$. Il est certain que des dieux tels que Trôsobios/Trzzubi et Tobaloas appartiennent à la seconde catégorie en tant que théoi agrioi, et que, d'autre part, le dieu pisido-lycien Kakasbos relèverait de la catégorie des dieux de la guerre. Comme dans le cas de Tlôs, la continuité louvito-lycienne est révélée aux IV et $v^{e}$ siècles av. J.-C, dans des inscriptions en langue lycienne trouvées dans des villes dont, pour certaines, l'existence est assurée au deuxième millénaire avant notre ère. Même si, sous l'effet d'une hellénisation de plus en plus forte, après le $\mathrm{IV}^{\mathrm{e}}$ siècle av. J.-C. la dénomination des dieux s'effectue généralement d'après un équivalent grec approximatif, il convient cependant de toujours reconnaître derrière l'habillage grec une personnalité divine authentiquement anatolienne; le cas est patent pour Artémis, les Dioscures, les Nymphes, Kronos ou encore les douze dieux ${ }^{6}$. Pour illustrer notre position, il suffira de passer en revue les dieux mentionnés dans les inscriptions en langue lycienne de quelques villes lyciennes significatives :

- Arneai : dans cette cité au nom typiquement anatolien ${ }^{7}$, le dieu de l'orage (Trqqa-/Trqqũt-) domine (TL 83, 15-16); en tant que divinité justicière, il est assisté de Maliya, déesse poliade de haute antiquité, protectrice des

Cf. R. Lebrun, Maliya, une divinité anatolienne mal connue, in Studia Paulo Naster oblata II, Louvain, 1982, p. 123-130.

5 Pour l'Artémis de Pergè, voir R. LEBrun, De quelques cultes lyciens et pamphyliens, in Festschrift S. Alp, Ankara, 1992, p. 357-361. Mentionnons, pour Komba, l'existence de nombreux autels dédiés à l'Artémis Kombikè. Quant à l'Artémis de Lagbè (Artémis Lagbènè), qu'il nous suffise de renvoyer à L. RoBert, Documents d'Asie Mineure, Paris, 1987, p. 438-441.

6 Pour Kronos (dont le culte n'est que peu attesté en Grèce), considéré comme le grand dieu de Tlôs, $c f$. L. RoberT, in $J S$ (1978), p. $43-48$ et ID., in $J S$ (1983), p. 249. La plupart des reliefs lyciens représentant les douze dieux doivent provenir de l'antique sanctuaire de Komba; au milieu des douze divinités on trouve aussi l'Artémis de Komba en tant que déesse chasseresse, régulatrice de la nature sauvage, de la faune, ainsi que parfois Hermès et le dieu «le Père », un dieu ancestral, dont le nom n'est jamais indiqué. Il convient de renvoyer sur ce sujet à la mise au point de L. RoberT, Documents d'Asie Mineure, Paris, 1987, p. 431-437, et je citerai la conclusion de l'article particulièrement pertinente : «..... dans ce culte des Douze dieux, autour des Douze, le plus souvent invoqués seuls, il y a un entourage éventuel, Artémis Chasseresse, le Père des Douze et aussi Hermès. Tous les reliefs viennent en définitive du même sanctuaire de Komba, mais il y a des variantes: les Douze sont normalement armés de l'épieu; sur le relief de Manisa, ils sont sans armes; ce sont pourtant les Dieux Chasseurs, car il y a les douze chiens-loups; une fois ils ont des boucliers. Les deux figures isolées sont Artémis et le Père, quelle que soit leur place. Là aussi nous touchons - comme pour les Dioscures et la Lune, pour Kakasbos aussi - au tuf religieux de ces régions, à leur caractère transmis du fond des âges et en dehors de toute influence hellénique. Là aussi les types ne sont pas imités de l'art et du culte grecs; la Grèce n'a fourni que les noms de la mythologie, Artémis, Hermès; même pas pour le Père, qui a gardé l'anonymat primitif ».

7 Arnéai : cf. L. Zgusta, Kleinasiatiscbe Ortsnamen. Heidelberg, 1984, p. 98, $997-2$. 
forces de la nature (TL 80). Il faut probablement encore mentionner le dieu Tobaloas pour cette période, bien que la seule mention connue à ce jour provienne d'un autel datable du II $^{\mathrm{e}}$ siècle ap. J.-C. où le dieu est qualifié de «bienveillant / qui exauce ${ }^{8}$.

- Antiphellos : dans les formules exécratoires des tombes rupestres de la ville, il n'est souvent fait mention que de l'ensemble des dieux : mabã $i$ buwedri. Toutefois, dans une inscription bilingue lycien-grec, la divinité justicière est la ẽni qlabi ebijebi « la mère du sanctuaire d'ici » correspondant à Létô dans la partie grecque du document. La longue inscription TL 55, difficile à comprendre car rédigée en lycien $\mathrm{B}$ toujours mal maîtrisé, accorde, elle, une place importante au dieu de l'orage Trqqñt?.

- Isinda : dans une inscription du $\mathrm{v}^{\mathrm{e}}$ siècle rédigée en lycien et en grec (TL 65), il est question de Trqqñt-/Trqqas auquel est associé, me semble-t-il, le dieu Ddeweze, en qui il faut peut-être reconnaître le nom lycien du Soleil hérité du louvite Tiwaza, comme j'ai essayé de le montrer dans une étude à paraître dans Hetbitica XIV. Dans les sources épigraphiques, les mentions ultérieures d'Artémis et des douze dieux renvoient certainement à un substrat anatolico-louvite: Artémis cache vraisemblablement une déesse protectrice de la nature sauvage, à moins que ce ne soit une divinité lunaire, tandis que les douze dieux constituent sans doute l'expression ramassée de l'ensemble des dieux « non supérieurs » de tout panthéon local anatolien.

- Kyaneai : la cité était célèbre grâce à un vieil oracle rendu par l'eau de la source, un oracle qu'Apollon s'appropria: il s'agit de l'Apollon Thyrxeus nommé en lycien turaXssali natri ${ }^{10}$.

- Limyra (lyc. Zêmuri): Les dieux de cette ville riche en tombeaux rupestres et en épigraphie funéraire nous sont connus en grande partie par les formules exécratoires. On y trouve la simple mention des dieux de la ville (TL 101, 4-5), mais aussi, de façon plus précise, la fameuse déesse ẽni qlabi ebijebi qui pourrait se dénommer Qebeliya d'après TL 102 , 4, et qui se voit parfois pourvue du qualificatif de pñtreñni, un adjectif ethnique dont le sens reste difficile à fixer ${ }^{11}$. Cette déesse justicière est normalement identique à la

\section{TAM II 758.}

9 Trqqñt semble être le seul théonyme intervenant dans l'inscription, avec trois mentions. Pour le sens de buwedri- " tout », $c f$. F. Sтавке, Untersucbung zur Stammbildung des keilscbriftluwiscben Nomens, Wiesbaden, 1990 (StBoT, 31), p. 467 sq., qui rapproche le terme lycien du louvite cunéiforme suwatar, susbtantif abstrait déverbatif de suwa(i)"remplir».

10 TL 44 c 47-48 (face C du pilier xanthien). Pour cet oracle, voir Paus., VII, 21, 13.

11 Ce terme qui qualifie parfois la «mère du sanctuaire d'ici » semble être un ethnique dont le sens reste difficile à préciser : ni le rapprochement du terme avec Pandaros (cf. G. Neumann, Beiträge zum Lykiscben V, in Die Sprache, 20, 2 [1974], p. 111), ni le rapprochement avec la ville de Patara (E. LAROCHE, in Fouilles de Xanthos, VI, Paris, 1979 , p. $75-76$ et 114) ne peuvent emporter l'adhésion. 
enni mabanabi "mère divine " signalée dans une inscription ${ }^{12}$. Nous connaissons aussi le dieu Trzzubi = grec Trôsobios dont le prêtre se fit bâtir un tombeau, ainsi que la divinité Qeli en qui G. Neumann voulut reconnaître l'aboutissement lycien du hittito-louvite Halki, la déesse Grain ${ }^{13}$. Enfin, il faut relever la mention probable des/du hppñterus mãhãi (TL 139, 3-4); s'agit-il dans ce cas d'un groupe divin justicier, comme ce fut proposé, ou n'a-t-on affaire qu'à un dieu ${ }^{14}$ ? Le culte ultérieur d'un Zeus asianique et des Dioscures nous assure de la pérennité de la tradition anatolienne. Quant au terme Xba- attesté dans l'inscription TL 102,4, il pourrait désigner le dieu Fleuve, en l'occurrence le Limyros, et le rapprochement avec la hourrite Hébat nous semble devoir être écarté ${ }^{15}$. J'attirerai encore l'attention sur une courte inscription bilingue trouvée au Létôon de Xanthos en 1966, faisant l'objet

\section{TL 134, 4}

13 Pour Trôsobios, cf. TL 111, où le prêtre du dieu se dénomme Erzesinube. Un prêtre de Qeli (lycien Qelebi kumaza) est mentionné dans l'inscription N 322 trouvée en 1974; pour le rapprochement avec Halki, voir G. NeUman, Namen und Epiklesen lykiscber Göttern, in Florilegium Anatolicum, Paris, 1979, p. 49; tout en retenant cette possibilité, R. Lebrun, Continuité cultuelle et religieuse en Asie Mineure, in Atti del II Congresso internazionale di Hittitologia, Pavie, 1995 (Studia Mediterranea, 9), p. 252-253, envisage aussi un rapprochement avec le louvite hali- « jour ».

14 Dans les deux attestations de hppñterus, ce mot précède mâhãi "dieux », mais il convient tout d'abord d'examiner si le terme est un nom. sing. ou un nom. plur.; dans l'inscription TL 58 (Antiphellos), le verbe qui doit exprimer la sanction est perdu : $5 \ldots . . .-]$ ili se hppñterus mâhâa mintehi, mais en TL 139, 4 le verbe est manifestement au singulier, ce qui implique aussi que la forme mâbãi = louvite massani- peut constituer un nom. sing, et pas uniquement un nom. pl. : 3.......................me ne 4 tubidi b [p] pîte [mus] mãhãa : se maraziya mintaba; de plus, mâhâa peut être aussi un génitif pluriel, et constituer ainsi un génitif partitif dépendant de bppñterus, qui, dans les deux cas, serait bien un nominatif singulier et pourrait signifier "représentant, ou haut fonctionnaire religieux, ou institution (religieuse) », à moins qu'il ne s'agisse d'un adjectif signifiant " grand, principal » ou un sens voisin; il ne peut s'agir d'un théonyme. $C f$. l'état du problème chez T.R. BRYCE, The Lycians in Literary and Epigrapbic Sources, Köbenhavn, 1986, p. 188.

15 Le rapprochement effectué par G. NEUMANN avec la grande déesse syro-hourrite Hébat du second millénaire me paraît devoir être écarté (in Festschrift Mansel, 1974, p. 640 sq.); en effet, la survivance d'un nom hourrite à l'époque gréco-asianique, de plus dans une région à l'époque très peu, voire non hourritisée, suscite le scepticisme. En revanche, reconnaître dans $X b a$ - la continuité du hittite-louvite hapa-/hapi- "rivière " constitue une piste d'autant plus que la trilingue xanthienne a fourni le thème verbal Xbai- « irriguer » : N 320, 14 : Xbaitẽ « ils irriguèrent », cf. louvite hapai-; l'anthroponyme gréco-lycien Kbamoas vient d'un étymon louvite Hapamuwa et non Hépamuwa comme on le croit parfois. Une information utile viendrait d'une interprétation correcte de $P d d e ̂ X b a-$, qui, en fin de compte, pourrait signifier «la rivière locale " et garder le souvenir de l'antique pratique de l'ordalie par le fleuve; la fin de l'inscription de Limyra TL 102 se comprendrait plus aisément, d'autant plus qu'il y est question de Qebeliya à laquelle le ou les coupable(s) doit/doivent payer l'amende.... : sewe: tubidi : $p d 4$ e $: X b a$ " et la rivière locale les frappera "., en l'occurrence le Limyros; rien ne me semble s'opposer à cette interprétation en $N 309$ c 6 , autre inscription de Limyra où $p d d e ̂ x b a$, manifestement noté comme un seul mot, se trouve entre trbbãmara et tesîmmi. 
d'une première étude de G. Neumann (Neufunde lykischer Inscbriften seit 1901, Wien, 1979, p. 29-30 = N 312), dont la lecture fut presque aussitôt améliorée par O. Carruba (Commento alle nuove Iscrizioni di Licia, in Festscbrift P. Meriggi I, Pavia, 1979, p. 79); elle se présente comme suit :
1. $\Delta \eta \mu \circ \lambda\left[\varepsilon^{\prime}\right] \delta \eta \varsigma[K \varepsilon \beta 1 \sigma \circ \varsigma$ ?]

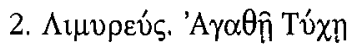

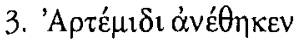
4. ñtemuXlida $k[e]$ bijeseb
5. zemuri ertemi
6. Xruwata [ubete ?]

Le texte lycien, négligeant la mention de la Bona Fortuna, se traduit comme suit : «Démokleidès, (fils) de K[é]biyésé a élévé une statue pour Artémis à Limyra ». Cette traduction voit dans Zemuri, non pas un ethnique, difficilement admissible du point de vue lycien mais qui correspondrait toutefois parfaitement au grec Limyreus, mais un locatif, ce qui soulignerait le culte d'Artémis (asianique) à Limyra.

- Myra : les inscriptions funéraires de la cette ville voisine de Limyra révèlent l'enracinement des dieux indigènes que sont Trqqas/Trqqñt-, le dieu de l'orage, Xba le «fleuve », Tesĩmi et sans doute Trbbãmara ${ }^{16}$. Plus tard, le nom de la déesse Éleuthéra, assimilée à Artémis, constitue en fait la traduction du gréco-anatolien Ereua $=$ hittite arawa " libre $»^{17}$. Tout aussi traditionnels sont les douze dieux, les Dioscures, Létô et les Nymphes.

- Rhodiapolis et Tyberissos : dans ces deux cités, la déesse dominante est sans conteste Maliya, la déesse poliade.

- Xanthos (lyc. Arñna) et le Létôon. : les documents en langue lycienne trouvés au Létôon prouvent le culte dans le sanctuaire de la êni qlabi ebijebi = Létô identifiée à une antique déesse de la source, des Eliyâna = les nymphes, de Maliya ainsi que de deux dieux cariens vénérés à Caunos (zone limitrophe avec la Lycie), à savoir Xñtawati Xbideñni = Basileus Kaunios, probablement le grand dieu de Caunos, et ArXXazuma $=$ grec Arkésimas ${ }^{18}$. La poursuite des fouilles nous apportera certainement de nouvelles indica-

16 Trbbañara: «interdiction», mot pouvant devenir une abstraction divinement personnifiée, $f f$. G. NeumanN, Neufunde lykischer Inschriften seit 1901, Wien, 1979, p. 25 : à décomposer en $\operatorname{trbb}(a)+$ mara « loi »; à la même page, l'auteur reconnaît en Tsm̃mi le nom de la déesse hittito-louvite Tasimi, Tesimi, lequel comporte encore de multiples variantes.

17 Cf. R. Lebrun, Problèmes de religion anatolienne, in Hethitica, 8 (1987), p. 246 et 256 ก. 12.

18 Pour les dieux vénérés à Xanthos et plus particulièrement au Létôon, $c f$. Chr. LE RoY, Aspects grecs et anatoliens des divinités vénérées au Létoon de Xanthos, in J. Borchhardt, G. Dobesch (éds), Akten des II. Internationalen Lykien-Symposions, Wien, 1993 (Ergänzungsbände zu den Tituli Asiae Minoris, 17), p. 241-247. 
tions. Les informations relatives au panthéon indigène de Xanthos même nous sont essentiellement fournies par la longue inscription du pilier xanthien (TL 44) dont la face D et la seconde partie de la face C sont plus difficiles à étudier du fait qu'elles sont rédigées en lycien B. De plus, dans certains passages encore obscurs des parties rédigées en lycien classique, la mention de théonymes n'implique pas nécessairement qu'ils furent vénérés à Xanthos; il peut être simplement fait allusion au culte de divinités de différentes cités lyciennes; le passage TL $44 \mathrm{C} 5-9$ est à cet égard significatif ${ }^{19}$. Néanmoins, nous pouvons affirmer l'émergence des cultes de Trqqas/Trqqñt-, de Maliya, des douze dieux, probablement de Xñtawati Xbideñni, culte caunien introduit dans la métropole lycienne, et de turaXssali Natri $=$ Apollon Thyrxeus.

- Je ne puis achever la revue de ces sites lyciens sans évoquer une inscription funéraire trouvée en 1966 à Kǐzĭlca près d'Elmalĭ par G. Bean; l'étude donnée par G. Neumann (op. cit. supra, p. 36-38 = N 314), a été reprise par O. Carruba (loc. cit. supra, p. 80-85). Particulièrement importante pour le culte de Kakasbos, elle se lit :

1. Xupãa 2. ebẽñni 3. menadẽ 4. masauwẽti 5. mejereb 6. tl ideimi 7. e] ti : Xñtawata 8. pl eriklebe (côté droit); 1. Xupaebebi 2. tijjadi: tike

3. zuñmẽ : tibe 4. mene tubidi 5. eti ebebẽ 6. XaXakba 7. 3 signes inconnus (côté gauche). : «Masaweti, [f]ils de Méjéré, a fait ce tombeau durant le gouvernement de Périclès (côté droit); celui qui fera quelque dommage à ce tombeau, Kakasbos le frappera en ce qui concerne ses ... (côté gauche), » L'intérêt de cette inscription est naturellement l'attestation du culte du dieu cavalier-guerrier Kakasbos en cet endroit, mais aussi de le montrer dans sa fonction de justicier protecteur de tombeaux et de nous donner le nom lycien de Kakasbos, lequel remonterait à un étymon anatolien *habatwa-, $c f$. hittite hattai- «frapper, abattre » (cf. Carruba, loc. cit. supra, p. 84-85); le

19 TL 44 C...... se 5 utãna : sttati : stala : ẽti: malijabl : pddãti 6 ddewẽ zXXazãi neu ne: meseweb :mmi: se Xbide 7 sttati mẽ : sttala: êti: qlabibijebi: se mal [i-] 8 jabi: se [x] ertemebi: se Xñtawatebi: Xbidêñ 9 bi. Selon la traduction proposée, des conclusions divergentes peuvent être tirées, le nœud du problème étant avant tout le sens précis du verbe stta- et, ensuite, le sens de Utãna dans lequel il me semble préférable, comme le suggère H.C. Melchert, Lycian Lexicon, Chapel Hill, $1993^{2}$ (Lexica Anatolica, 1), p. 85, d'y voir le toponyme Hytenna, plutôt que l'anthroponyme Otanès. Quant au verbe stta-, il constitue un verbe intransitif « être debout, se dresser » (cf. latin stat) car par deux fois dans le passage analysé ici, sttala est bien un nominatif sujet de stta-, et non un accusatif objet direct du verbe en question. La traduction du début du passage serait donc : « ... et 5 à Hytenna se dresse une stèle dans le temenos de Maliya 6 en face des combattants..... et à Caunos 7 se dresse une stèle dans le sanctuaire d'ici ? et de Mal [i] ya 8 et d'Artémis et de Basileus Caunos.. ». En fait, nous avons ici une information sur un culte de la cité lycienne d'Hytenna (cf. L. ZGusta, Kleinasiatiscbe Ortsnamen, Heidelberg, 1984, p. $656 \S$ 1417) et d'autres indications cultuelles relatives à Caunos, mais rien ne concerne spécifiquement Xanthos. Ce passage est interprété différemment, notamment en reconnaissant dans Utana l'anthroponyme Otanès et en concluant à la mention de cultes xanthiens, par R. Gusmani, Su due termini della Trilingue di Xanthos, in Festscbrift P. Meriggi I, Pavia, 1979 (Studia Mediterranea, 1), p. 227-229. 
théonyme lycien supprime tout rapprochement, néanmoins tentant, avec le louvito-lycien asba-lesbe- " cheval ».

Cet examen rapide n'a concerné que quelques villes lyciennes, mais celles-ci nous paraissaient fournir une documentation significative du maintien des traditions louvito-lyciennes en plusieurs coins de Lycie aux $\mathrm{v}^{\mathrm{e}}$ et $\mathrm{IV}^{\mathrm{e}}$ siècles av. J.-C. face à une hellénisation de plus en plus forte, mais qui ne parviendra pas à étouffer complètement le tuf́r religieux anatolien. De façon générale, Trqqñt, le dieu de l'orage, l'êni qlabi ebijebi / ẽni mabanabi à laquelle s'assimilera Létô, soit la source fécondante, avec son cortège des Eliyãna = les nymphes, et Maliya, déesse poliade protégeant les champs et les vergers, s'imposent presque partout; la personnalité de ces dieux subsistera même si plus tard ils ne seront désignés que sous un vocable grec. Inversément, des divinités connues à la période gréco-romaine uniquement par leur appellation grecque renvoient à une personnalité anatolienne dont le nom n'est pas toujours connu à l'heure présente : c'est le cas de diverses Artémis, d'Apollon ou des Dioscures.

E. Dans le Sud-Est anatolien, le long de la côte méditerranéenne, se situe Kôrykos, un important centre religieux cilicien de l'époque gréco-romaine, dont, toutefois, le nom au deuxième millénaire avant $J$.-C. nous demeure inconnu. La nature des croyances et du culte révèle la haute antiquité de ce foyer religieux; de plus, il convient de prendre en compte dans toute étude du site non seulement le sanctuaire de la ville, mais aussi dans son voisinage immédiat, l'antre corycien, lieu de résidence célèbre du monstre Typhée et prison momentanée de Zeus vaincu par Typhée qui lui avait de plus sectionné les nerfs et caché ceux-ci dans une outre en un lieu secret; Zeus fut cependant miraculeusement délivré par Pan et put reprendre le combat contre le monstre cilicien dont il finit par triompher. Tout cet épisode mythique trouve son origine dans le mythe hittite opposant le dieu de l'orage Tarchunt au dragon Illuyanka ${ }^{20}$. Nous constatons ici un lien immédiat avec les traditions mythiques et religieuses du deuxième millénaire. Dans la ville même de Kôrykos étaient vénérés principalement Hermès, Zeus, Pan et les nymphes ${ }^{21}$. L'anthroponymie était, comme dans la ville voisine d'Olba, majoritairement d'origine louvite et comportait de nombreux noms théophores $^{22}$. À l'époque gréco-romaine, Kôrykos apparaît comme la cité du dieu

20 Voir les lignes de F. VIAN, Nonnos de Panopolis, Les Dionysiaques chants I et II, Paris, 1976, p. 25-26 de la notice; Ph. Houwink TEN CATE, The Luwian Population Groups of Lycia and Cilicia Aspera during the Hellenistic Period, Leiden, 1961, p. 206-212.

21 Oppien, lequel vécut à Kôrykos, désignait cette cité comme la ville d'Hermès, cf. Oppien, Hal., III 7-8, 208-209 et les remarques du scholiaste sur III, 207.

22 Cf. HOUWINK TEN CATE, op. cit. (n. 20), p. 202-205; Olba, à identifier peut-être avec la ville louvito-hittite de Ura, adorait particulièrement Zeus (le Zeus Olbios constituait sans doute l'avatar de l'antique Tarcbunt de Ura); une liste de prêtres (I ${ }^{\text {er }}$ siècle av. J.-C.) et du collège de rhabdouques particulièrement significative pour la survie d'anthroponymes louvites a été publiée et étudiée par G. Dagron et D. Ferssel, Inscriptions de Cilicie, Paris, 
3Hermès, mais dans ce nom, tout comme dans celui de Zeus, des nymphes, voire de Pan, il faut reconnaître l'habillage, le déguisement grec de dieux louvites. En effet, comme l'a bien montré notre collègue Ph. Houwink ten Cate, Hermès est à identifier au dieu louvite Kurunta/Runta, soit un des fameux dieux notés fréquemment à l'aide du sumérogramme lamma dans les textes hittito-louvites, ces divinités protectrices de la nature sauvage, dont, comme à Kôrykos, le culte se perpétuera jusqu'à l'époque gréco-romaine ${ }^{23}$.

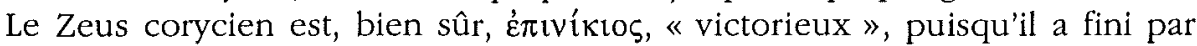
triompher de son adversaire Typhée, tout comme l'on racontait au deuxième millénaire que le dieu de l'orage louvite avait vaincu le dragon Illuyanka.

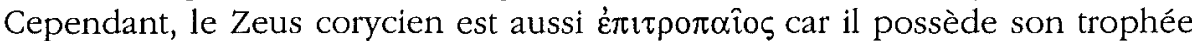

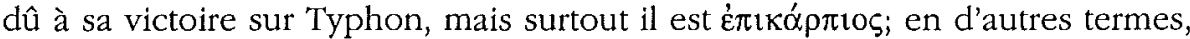
comme le Zeus Tarsios, il est aussi un dieu du blé, de la vigne, des fruits, donc un dieu de l'orage qui n'est pas uniquement le dieu terrifiant de la foudre, mais également un dieu qui apporte l'abondance grâce à la pluie bienfaisante. En cela, il perpétue l'image du Tarchunt louvite telle qu'elle est connue notamment par des textes et plusieurs reliefs ciliciens du premier millénaire avant notre ère; ainsi, les reliefs néo-louvites d'Ivriz, de Bor, une stèle à l'entrée du musée de Niğde, les textes de Kululu ou de Sultan Han ${ }^{24}$.

F. Restant au bord du littoral cilicien, on se doit de citer le cas de la ville de Tarse comme étant un lieu illustratif de la continuité cultuelle. Cette ville, dont le prestige intellectuel était grand à l'époque de saint Paul, pouvait se prévaloir d'une très haute antiquité, ce que confirment les fouilles non seulement au niveau de la préhistoire, mais encore pour la période hittite. En effet, les fouilles - dont on regrettera l'interruption - entreprises par Miss H. Goldmann ont révélé de nombreux vestiges hittites de toute première importance : bâtiments, rues, sceaux, tablettes; il est d'ailleurs surprenant que les archives hittites de Boghazköy-Hattusa, bien que mentionnant la ville cilicienne de Tarsa, n'aient pas encore livré plus d'informations sur la ville. Quoi qu'il en soit, Tarse au deuxième millénaire comportait certainement deux grandes figures divines : le dieu de l'orage maniant la foudre, mais aussi porteur de l'épi de blé et de la grappe de raisin que nous retrouvons bien figuré sur les monnaies à l'effigie de Zeus/Baal Tarsios, mais aussi le dieu Santa, une

1987 (Travaux et mémoires du Centre de recherche d'bistoire et civilisation de Byzance, Collège de France, Monograpbies, 4), , p.27-37. Ura se trouvait en tous cas dans la région de Silifke, cf. A. Lemaire, L'écriture pbénicienne en Cilicie, in Pboinikeia Grammata, Namur, 1991 (Coll. d'Études Classiques, 6), p. 138 et n. 19.

23 Cf. Houwink ten Cate, op. cit. (n. 20), p. 212-213; Dagron - Feissel, op. cit. (n. 22), p. $45-46$.

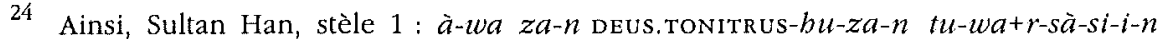
ta4-nu-wa-ba: et j'ai installé le dieu de l'orage (Tarchunt) de la vigne que voici »; stèle

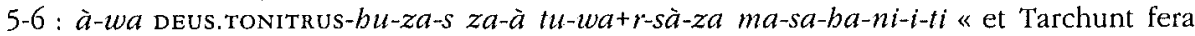
croître les vignes que voici »; Tarbuzas tuwarsasis (nominatif) est la désignation type de ce dieu de l'orage à la vigne. 
divinité souvent redoutable, à l'aspect guerrier et susceptible d'engendrer de nombreux fléaux, qu'il y a tout lieu de reconnaître dans le dieu Sandôn assimilé à Héraklès ${ }^{25}$. De futures études basées sur l'élargissement de la documentation contribueront peut-être à souligner le caractère indigène d'autres dieux liés selon certains auteurs anciens, tels Dion de Pruse, à la ville de Tarse.

G. Pour terminer notre réflexion, réservons une place à Comana Cappadociae / Hierapolis, située le long du Saros, dont les premiers vestiges furent dégagés et étudiés en 1881, ce qui aboutit à une première publication des inscriptions par Waddington en $1887^{26}$. Après une longue interruption, les fouilles ont repris voici quelque trente ans sous l'égide du British Institute of Archaeology à Ankara ${ }^{27}$. L'histoire religieuse de ce site prend un relief tout particulier si l'on admet que notre Comana est à identifier avec Kummanni, un des plus grands centres religieux du monde hittite, en plein pays kizzuwatnien $^{28}$; Kummanni, fortement marquée par les conceptions religieuses hourrites ( $c f$. les idées des théologiens d'Alep), joua un rôle déterminant dans le processus de la hourritisation du panthéon impérial et des sphères sociales dominantes dans la capitale Hattusa. On imagine l'importance que revêtira la mise à jour des vestiges - notamment des bibliothèques - de la Comana du deuxième millénaire avant notre ère. À cette époque, et plus précisément avant l'annexion du royaume de Kizzuwatna à l'État hittite, les dieux principaux de la ville, essentiellement hourrites, sont Teshub et sa parèdre Hébat, Shaushka, Ea et Kubaba, la grande déesse de Kargémish, qui y fera son éntrée sans doute après les campagnes des rois hittites en Syrie ( $\mathrm{XvI}^{\mathrm{e}}-\mathrm{x} \mathrm{v}^{\mathrm{e}}$ siècle av. J.C.). Sarrumma, dieu montagne cilicien, devenu au Kizzuwatna d'abord, et ensuite dans le cadre du panthéon impérial tardif, le fils du couple TeshubHébat, n'est curieusement pas attesté à Kummanni alors que seṣ centres cultuels, à savoir Laiuna, Uda et Urikina se trouvent au Kizzuwatna, que de plus Sarrumma est représenté en roi de la montagne sur le relief de Hanyeri à quelque distance de Comana et qu'enfin le culte de Sarrumma est bien attesté dans les inscriptions néo-louvites de Cilicie. Quelques éléments de son culte et de sa personnalité devaient subsister en Ciìicie à la période gréco-romaine, mais nous ignorons derrière quel théonyrne grec précis se cachait Sarrumma à cette époque, tandis qu'il nous faut cependant constater

25 E. LARoche, Un syncrétisme gréco-anatolien: Sandas = Héraklès, in Les syncrétismes dans les religions grecque et romaine, Paris, 1973, p. 103-114.

26 W.H. Waddington, Inscriptions de la Cataonie, in $B C H, 7$ (1883), p. 125-148.

27 R.P. Harper, I. Bayburtluoglu, Preliminary Report on Excavations at Sar, Comana Cappadociae in 1967, in AnSt., 18 (1968), p. 149-158. Pour les inscriptions trouvées à cette occasion, $c f$. R.P. HARPER, Inscriptiones Comanis Cappadociae in A.D. 1967 effossae, in AnSt, 19 (1969), p. 27-41.

28 Voir H.M. KüMMEL, Kummanni, in RlAss. 6 Band (1980-1983), p. 335-336. 
l'abondance des noms théophores de sarma ${ }^{29}$. En ce qui concerne précisément Kummanni, une absence, peut-être provisoire, d'informations existe pour la période s'étalant de la fin du deuxième millénaire à la période grécoasianique. Dès cette époque, les choses s'éclaircissent pour l'histoire religieuse de la cité. De bons renseignements sont dus à la plume de Strabon : «Dans le massif de l'Anti-Taurus se trouvent des vallées étroites et profondes. C'est là que sont situés Comana et le sanctuaire d'Enyô que ceux-ci appellent (sanctuaire) de Mâ. Comana est une ville considérable, mais sa population se compose en majeure partie des théophorètes et des serviteurs du temple.... Le prêtre est le maître du sanctuaire et des serviteurs du temple, qui étaient plus de 6000 à l'époque où je fis le voyage de Comana, hommes et femmes. Du sanctuaire relève un territoire très étendu dont les revenus vont au prêtre. Aussi, celui-ci tient-il en Cappadoce le deuxième rang après le roi ${ }^{30}$. En confrontant les sources écrites anatoliennes du deuxième millénaire avec ce passage de Strabon, on ne peut s'empêcher d'établir une solution de continuité entre l'ancienne Kummanni et la Comana grécoromaine : retenons l'importance cultuelle de la cité, véritable ville sainte, la position sociale éminente du grand-prêtre, les classifications sacerdotales, les possessions et revenus du temple, enfin le nom même de la grande déesse $M a \hat{a}$, un théonyme manifestement anatolien ${ }^{31}$, qui ne nous aide cependant pas à définir la personnalité de la déesse. Sa représentation sur des monnaies et sur. un relief étudié par $H$. Seyrig montre une déesse radiée, éventuellement, comme sur le relief, entourée de deux aigles juchés sur ses épaules; la tête de la déesse nimbée de rayons, l'association d'aigles, messagers du Soleil, à sa personnalité inviteraient à y reconnaître une divinité solaire ${ }^{32}$. D'autre part, son association à Enyô en ferait une divinité guerrière. Dès lors, l'anatolienne Mâ de l'époque gréco-romaine n'illustrerait-elle pas un retour à une très ancienne divinité de la cité, déesse reine et guerrière, assimilée à Hébat devenue la grande déesse de la ville, dont elle récupère le caractère solaire? Avec l'affaiblisement, voire la disparition de la culture hourrite, à Kummanni comme en d'autres lieux de Cilicie, on en serait revenu à la dénomination pré-hourrite de la grande déesse de la ville, dont nous découvrons le nom à une période tardive; une telle situation est patente dans le cas du dieu de l'orage où au premier millénaire Teshub cède la place à Tarchunt. À titre informatif, en fonction de la documentation épigraphique réunie, nous signalerons aussi l'attestation des cultes de la très grande déesse $=\Theta \varepsilon \dot{\alpha} \mu \varepsilon \gamma \hat{i} \sigma \tau \eta$, de Kúpros 'A $\pi$ ó $\lambda \lambda \omega v=$ Apollon cataonien dont il était fait le plus grand cas,

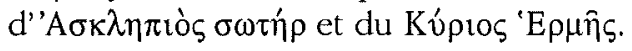

29 Cf. Houwink ten Cate, op. cit. (n. 20), p. 134-136; E. Laroche, Le diell anatolien Sarruma, in Syria, 40 (1963), p. 301-302.

30 Strabon, XII, $2,3$.

31 Mâ pourrait constituer l'haplologie du nóm divin Mamma bien attesté dans les textes hittites; voir aussi $\mathrm{M} \alpha(\varsigma)$ dans l'épigraphie gréco-asianique.

32 H. Serrig, Scripta Varia, Paris, 1985, p. 705-708. 
Les quelques exemples ainsi réunis de l'Ouest à l'Est de l'Anatolie méditerranéenne suffisent, me semble-t-il, à donner l'orientation des recherches futures. Nous observons qu'en dépit d'une hellénisation de plus en plus appuyée, en des lieux de grande tradition religieuse et de plus parfois d'accès difficile à cause du relief, les résistances cultuelles se sont imposées et restent un fait quotidien encore au début de notre ère, même si le nom divin se note en grec. Toutefois, l'inventaire (provisoire) des noms divins indigènes en Lycie donne à penser que l'hellénisation progressa plus facilement dans la partie occidentale de celle-ci ${ }^{33}$.

Université Catholique de Louvain

René LEBRUN

Institut Orientaliste, Collège Erasme,

Place Blaise Pascal, 1

B - 1348 Louvain-LA-Neuve

\&

Institut Catholique de Paris

E.L.C.O.A.

21, rue d'Assas

F - 75270 Paris Cedex 06

33 Ed. Frezouls, Les cultes de la Lycie occidentale, in J. BorchHardt, G. Dobesch (éds), Akten des II. Internationalen Lykien-Symposions, Wien, 1993, p. 203-212. 